\title{
Effect of a urinary catheter on seed position and rectal and bladder doses in CT-based post-implant dosimetry for prostate cancer brachytherapy
}

\author{
Hiroaki Kunogi, MD, PhD', Nanae Yamaguchi, MD', Yoshiaki Wakumoto, MD, PhD², Keisuke Sasai, MD, PhD' \\ 'Department of Radiation Oncology, ${ }^{2}$ Department of Urology, Juntendo University, Hongo, Bunkyo-ku, Tokyo, Japan
}

\begin{abstract}
Purpose: To assess the variability in rectal and bladder dosimetric parameters determined according to post-implant computed tomography (CT) images in patients with or without a urethral catheter.

Material and methods: Patients with prostate cancer who were scheduled to undergo CT after brachytherapy between October 2012 and January 2014 were included. We obtained CT series with and without a urinary catheter in each patient. We compared the rectal and bladder doses in 18 patients on each CT series.

Results: The shifts in the seed positions between with and without a catheter in place were $1.3 \pm 0.3 \mathrm{~mm}$ (mean \pm standard deviation). The radiation doses to the rectum, as determined on the CT series, with a urethral catheter were higher than those on CT without a catheter $(p<0.001)$. Radiation doses to the bladder with a catheter were significantly lower than those without a catheter $(p=0.027)$.

Conclusions: Post-implant dosimetry (PID) with no catheter showed significantly lower rectal doses and higher bladder doses than those of PID with a catheter. We recommend the PID procedure for CT images in patients without a catheter. Use of CT with a catheter is limited to identifying urethral position.
\end{abstract}

Key words: brachytherapy, CT-based, prostate cancer, rectal dose, urinary catheter.

\section{Purpose}

Brachytherapy is an effective treatment option among radiation therapies. Permanent prostate brachytherapy using ${ }^{125} \mathrm{I}$ achieves good results in patients with low- or intermediate-risk prostate cancers [1-4]. Combination therapies of external beam radiation therapy (EBRT) and brachytherapy appear superior for high-risk patients, compared with more localized treatments, such as surgery alone or EBRT [3,5]. Therefore, seed-implant brachytherapy is often selected not only for low- and intermediate-risk but also high-risk prostate cancers.

Post-implant dosimetry (PID) of seed brachytherapy is essential to evaluate the quality of prostate radioactive seed implantation [6] and must be performed in all patients undergoing these treatments [7]. Computed tomography (CT)-based PID is the most commonly used method for dosimetric assessment [8], as it enables an evaluation of seed placement accuracy, actual target coverage, and the radiation dose to other risk organs [8-10]. Although contouring the rectal and bladder outer walls is easy on CT, the urethra is difficult to identify and to contour on CT-based PID [11]. Stock et al. used a Foley catheter to define the urethral po- sition for PID [12]. The American Association of Physicists in Medicine (AAPM) recommends optional use of a Foley catheter for the post-implant urethral dose evaluation [13]. Crook et al. also recommended that the urethra should be contoured as a structure on each slice where seeds can be seen, and that the urethra should be identified by either catheterization or fusion by transrectal ultrasound [14].

A patient with prostate cancer and radioactive seeds is irradiated without a urinary catheter in place after implantation. However, the post-implant setting is different from the actual state of irradiation. Ohashi et al. assessed the variability in dosimetric parameters with postural changes on PID [15]. The post-implant setting with a urinary catheter is also different from the actual state of irradiation and might not represent the precise dose distribution to risk organs such as the rectum and optimized intraprostatic sector dosimetric quality [16]. Post-implant dosimetry quality is unsatisfactory without accurate post-implant analysis for organs at risk [8,17-20]. There are few reports evaluating the changes of seed distribution on CT images and the dosimetric differences for the rectum and bladder doses, as a result of using a urinary catheter during PID. The rectal dose needs particu- 
larly accurate evaluation during permanent prostate brachytherapy combined with EBRT to adjust the EBRT dose distribution and avoid excess morbidity.

We investigated the shift in sources and the difference in rectal and bladder doses between with and without a urinary catheter in place on CT-based PID in this study. This study was approved by the Ethics Board of our institution.

\section{Material and methods \\ Permanent prostate brachytherapy}

Eighteen patients with low-risk prostate carcinoma (T1-2a, prostate specific antigen $<10 \mathrm{ng} / \mathrm{ml}$ and Gleason score $\leq$ 6) between October 2012 and January 2014 underwent transperineal interstitial permanent prostatic implantation (monotherapy) using loose ${ }^{125} \mathrm{I}$ radioactive seeds (Oncoseed 6711, GE Healthcare Medi-Physics Inc., Arlington Heights, IL, USA or STM1251, C.R. Bard, Covington, GA) at our institution. Mean activity per seed was $0.403 \mathrm{U}$ (range, 0.361-0.502 U). Signed informed consent was obtained from each patient prior to seed implantation. The planning target volume (PTV) was defined as the entire prostate. The dose prescribed to the PTV during intraoperative planning was 145 Gy. The sources were loaded using a Mick applicator (Mick Radio-Nuclear Instruments Inc., Mount Vernon, NY, USA), and Variseed ver. 8.0 (Varian Medical Systems Inc., Palo Alto, CA, USA) software was used to determine the PID.

\section{Post-implant image acquisition}

Computed tomography data were acquired 4 weeks after permanent prostate brachytherapy for the dosimetric analysis according to the recommended schedule [21]. Post-implant CT was performed using a spiral CT (HiSpeed Dxi; GE Healthcare, Buckinghamshire, UK). Computed tomography scanning was performed in patients in the supine position with a urinary catheter $(8 \mathrm{Fr}, 2.7 \mathrm{~mm})$ in place, and a field of view (FOV) of $50 \mathrm{~mm}$ and the same pitch of $2 \mathrm{~mm} / 2 \mathrm{~mm}=1$ (defined as the table feed/total detector width of the collimated beam). The urinary catheter was withdrawn with great care after the first scan to prevent perturbing the patient, and a second scan was successively performed in the same position without the catheter in place and using the same FOV. No intravenous contrast material was used.

\section{Fusion method}

The CT scans with a catheter can be optimally matched to additional CT scans without a catheter because these images were obtained simultaneously from the same position using the same FOV. After the first scan, the urinary catheter was withdrawn with great care to avoid disturbing the patient.

\section{Contouring the prostate, urethra, rectum, and bladder and identifying seed source positions with and without a urinary catheter in place}

The prostate was contoured with and without a urinary catheter. The $\mathrm{D}_{90}$ (i.e., minimum dose received by
$90 \%$ of the prostate volume expressed as a percentage of the prescription dose) and $V_{100}$ values (i.e., percentage of the prostate volume covered by $100 \%$ of the prescription dose) were estimated to verify the validity of the prostate dose coverage in this study. The prostatic urethra was contoured with and without a urinary catheter. The urethra on PID with the catheter was contoured as a circular structure $4 \mathrm{~mm}$ in diameter based on a urinary catheter position from the prostatic base to the apex (Figure 1), and the urethra on PID without the catheter was contoured as a circular structure of the same diameter $(4 \mathrm{~mm})$ by adequately adjusting the position of the urethra on fusing CT images with the catheter considering the shift of the seeds located close to the catheter.

To estimate the effects of a urethral catheter on the rectal/bladder doses and on source positions, the rectal and bladder outer walls on CT were contoured with or without the urinary catheter, and seed source positions with or without the urinary catheter were identified as the bright position on CT using Variseed ver. 8.0 software. The automatic seed finding function with manual adjustments was used in the source reconstruction procedure. We automatically and completely fused the two sets of CT images (with and without a catheter) because patients remained in the same position during the CT studies, and we ensured that the time interval between the two sets of CT images was very short. We confirmed almost no difference in pelvic bone position using the image blending function in Variseed ver. 8.0. The outline of the rectum was finely adjusted if there was a slight difference in rectal position between each set of CT images due to rectal peristaltic motion. That of the bladder was contoured again because bladder position seemed to be affected slightly by the urinary catheter.

\section{Evaluation of source position shifts and dosimet- ric parameters with versus without a urinary cath- eter in place}

We measured the source shift in the 1,200 three-dimensional coordinate positions $(x, y, z)$ on the three-dimensional spaces in the 18 patients with and without a urethral catheter on both CT series using VariSeed Source Location Export in Variseed 8.0 software.

We compared each $D_{90}$ value (i.e., the minimum dose received by $90 \%$ of the prostate volume), each $V_{100}$ value (i.e., the percent volume of the post-implant prostate receiving $100 \%$ of the prescribed dose), each $\mathrm{UD}_{10}$ and $\mathrm{UD}_{30}$ value (i.e., minimum doses received by $10 \%$ and $30 \%$ of the urethral volume, respectively), each $\mathrm{RD}_{2 \mathrm{cc}}$ value (i.e., the minimum dose received by $2 \mathrm{cc}$ of the rectum), and each $\mathrm{RV}_{100}$ value (i.e., the rectal volume receiving > 100\% of the prescription dose) to evaluate the calculated dosimetric parameters, as recommended by the AAPM Task Group 137 [13]. In addition, we compared each $\mathrm{BD}_{1 \mathrm{cc}}$ value (i.e., minimum dose received by $1 \mathrm{cc}$ of the bladder) as a dosimetric parameter for the bladder.

\section{Statistics}

Each data point was compared using the paired-sample $t$-test to assess the estimated prostate volumes and re- 


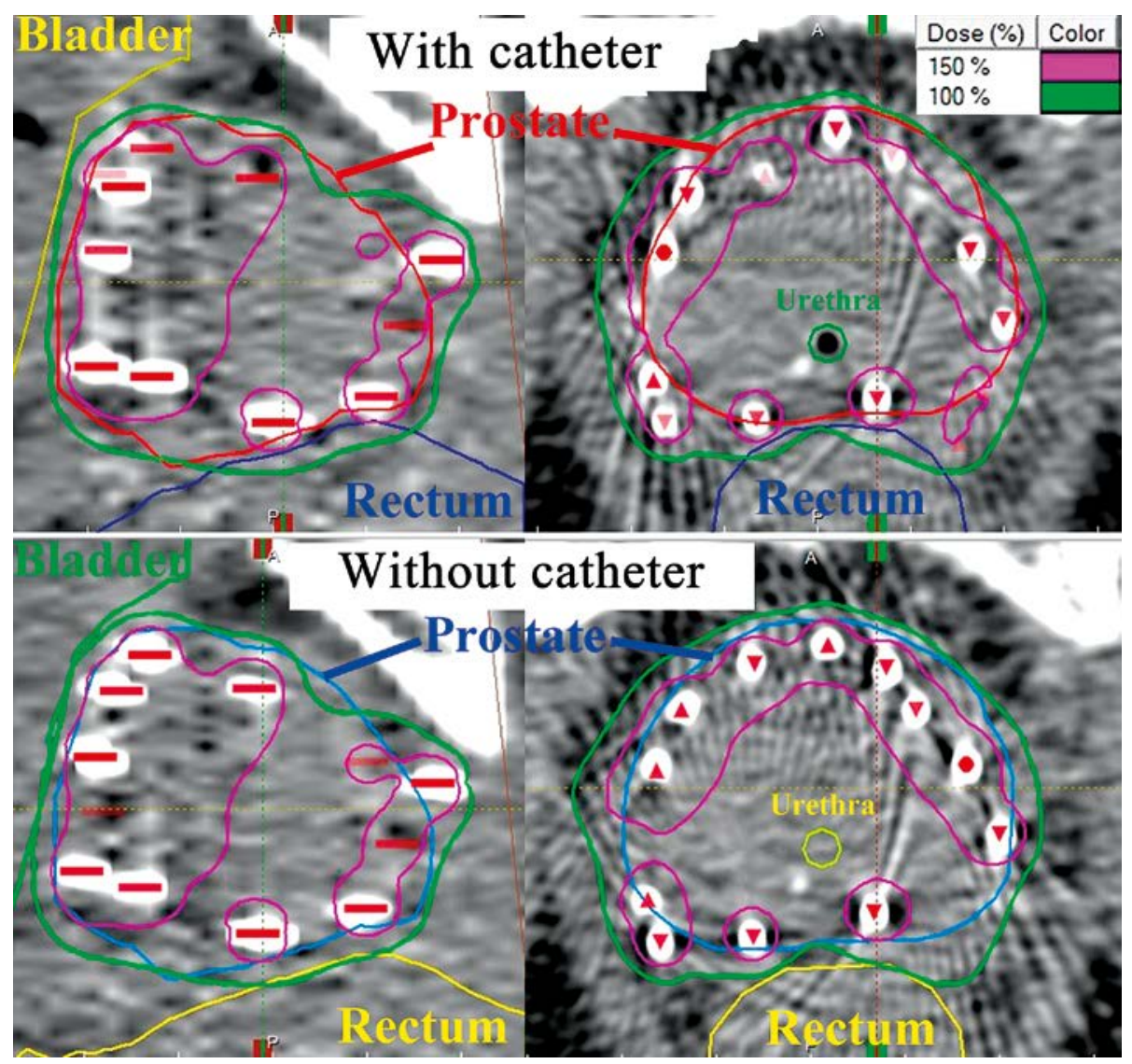

Fig. 1. Example of isodose distributions in the sagittal plane (left) and transverse plane (right) on each PID. In the two upper images, the prostate (red), urethra (green), bladder (yellow), and rectum (dark blue) are contoured on CT images with a catheter. On the two lower images, the prostate (blue), urethra (lemon), bladder (light green), and rectum (light yellow) are contoured on CT images without a catheter. Each red source image indicates each seed source position (the bright image) on each CT image. The seeds move with the target, and a shift in the dose distribution is apparent. The overlay of the $100 \%$ isodose area and rectum volume on PID without a catheter is less than that on PID with a catheter

sults of the analysis of dose-volume histograms (DVHs) with versus without a urinary catheter on CT. Analyses were performed using SPSS ver. 18 (SPSS Inc., Chicago, IL, USA). $P$-values $<0.05$ were considered significant.

\section{Results}

The mean source location difference in 1,200 sources among 18 patients between the CT series with and without a catheter in place was $1.3 \mathrm{~mm}$ (standard deviation (SD): $0.3 \mathrm{~mm}$ ). Figures 1 and 2 show the displacements in the source positions and dose distributions, respectively. The estimated prostate volumes and results of the analysis of dose-volume histograms (DVHs) were compared with and without the catheter (Table 1). The $\mathrm{D}_{90}$ and $\mathrm{V}_{100}$ values for the post-implant prostate in all 18 patients were significantly different on CT with versus without the catheter $\left(\mathrm{D}_{90}\right.$ (mean $\left.\pm \mathrm{SD}\right): 164(95 \%) \pm 20$ Gy vs. $162(94 \%) \pm 20 \mathrm{~Gy}$, $p=0.012 ; \mathrm{V}_{100}$ (mean $\left.\pm \mathrm{SD}\right): 113 \pm 14 \%$ vs. $111 \pm 14 \%$, $p=0.023)$. The $\mathrm{UD}_{10}$ and $\mathrm{UD}_{30}$ values in 18 patients were significantly different on CT with versus without the catheter $\left(\mathrm{UD}_{10}\right.$ (mean $\left.\pm \mathrm{SD}\right): 152 \pm 19 \%$ vs. $157 \pm 19 \%, p<0.001$; $\mathrm{UD}_{30}($ mean $\pm \mathrm{SD}): 139 \pm 14 \%$ vs. $\left.143 \pm 13 \%, p=0.001\right)$.
The $\mathrm{RD}_{2 \mathrm{cc}}$ and $R V_{100}$ values in all 18 patients were significantly different on the CT series with vs. without the catheter $\left(\mathrm{RD}_{2 \mathrm{cc}}\right.$ (mean $\left.\pm \mathrm{SD}\right): 93.4 \pm 13.9 \mathrm{~Gy}$ vs. $85.1 \pm 11.9 \mathrm{~Gy}$, $p<0.001 ; \mathrm{RV}_{100}$ (mean $\pm \mathrm{SD}$ ): $0.42 \pm 0.32$ cc vs. $0.21 \pm 0.26 \mathrm{cc}$, $p<0.001)$. $\mathrm{BD}_{1 \mathrm{cc}}$ values determined on the $\mathrm{CT}$ series were significantly lower with a catheter than those without the catheter in place $\left(\mathrm{BD}_{1 \mathrm{cc}}\right.$ (mean $\left.\pm \mathrm{SD}\right)$ : $75.3 \pm 17.1 \mathrm{~Gy}$ vs. $81.2 \pm 23.2 \mathrm{~Gy}, p=0.027)$. Figure 3 shows the $\mathrm{RD}_{2 \mathrm{cc}}$ and $\mathrm{BD}_{1 \mathrm{cc}}$ values of each patient.

\section{Discussion}

Post-implant dosimetry without a catheter showed significantly lower rectal doses and higher bladder doses than PID with a catheter (Figure 3). Post-implant dosimetry without a catheter demonstrated a state closer to an actual state of irradiation (comprising the source positions, the prostate, organs at risk, and a dose distribution). We invented a new two-step PID technique using the two sets of CT images (with and without a catheter) for accurate DVH assessment. After the first CT scanning with a urinary catheter, the urinary catheter must be withdrawn with great care, and the second scan with- 


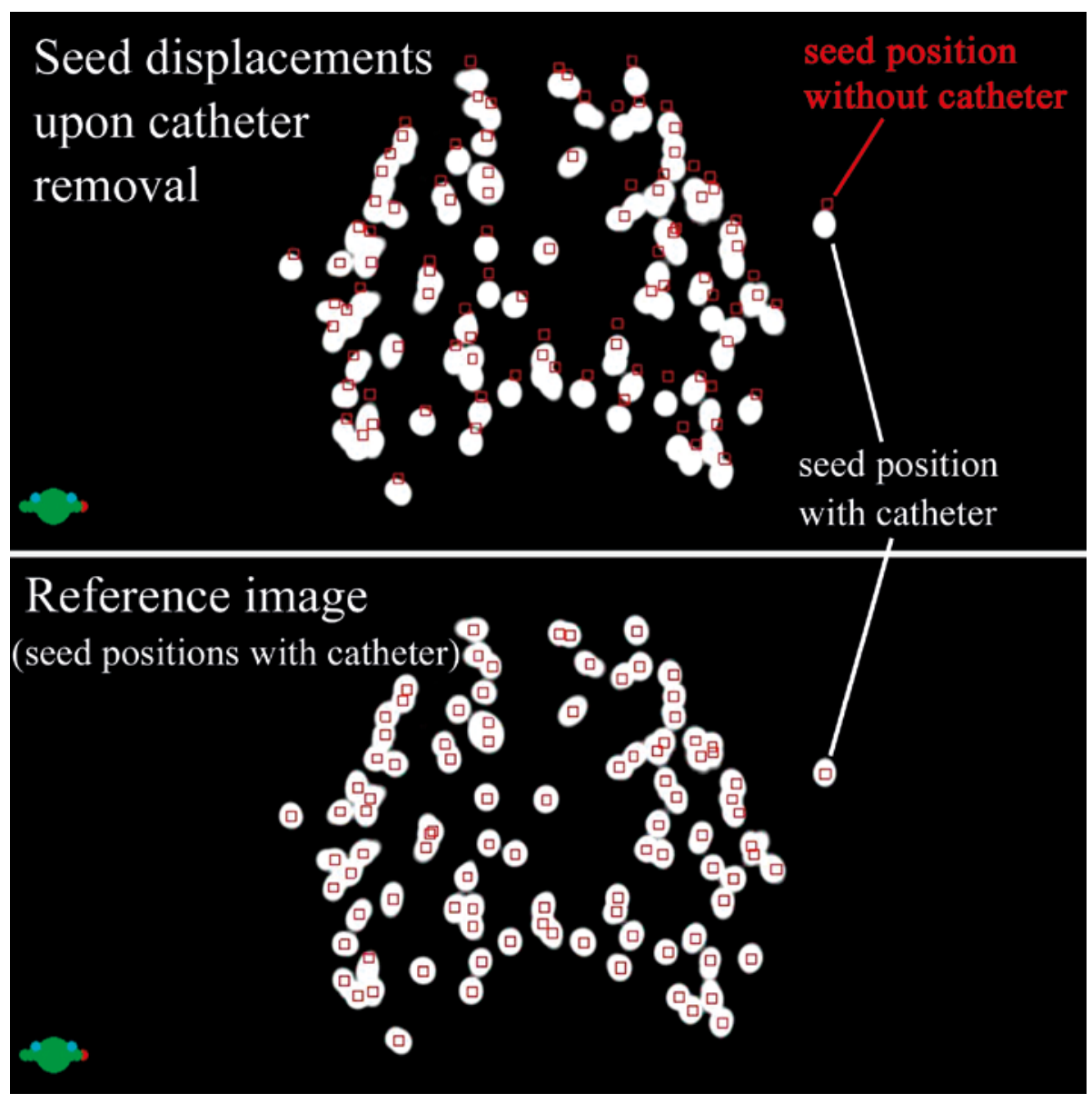

Fig. 2. Projection image of the source in the transverse dimension. In the upper image, the red source images are seed source positions without a catheter, and the bright images are source positions with the catheter. The shifts on the ventrodorsal and crosswise directions are apparent in this image. The lower image is the reference image as a comparison with the upper image to understand the source shift. In the lower image, the red source images and bright images are seed source positions with a catheter

out the catheter must be successively performed in the same position. In the first step, PID will be performed by CT images without the catheter, except for the identification of the urethra. In the next step, we can identify the urethral position using CT with the catheter, and this position could be accurately matched to $\mathrm{CT}$ without the catheter used in the first step because both CT images are co-registered. The CT images without a catheter are used to determine accurate dose distributions, whereas the fusing CT series with a catheter is used to identify the urethral position. This technique using the two sets of CT images will provide high-quality PID. In this procedure, the urethra position obtained from the fusing CT images with a catheter is not always adequate because the urethra position changes when a catheter is inserted into the urethra. The displacements of seed positions located close to the catheter will reveal residual displacements of the urethra position obtained from the fusing CT images with a catheter. The urethra position obtained from the fusing CT images with a catheter may require correction.

Post-implant dosimetry without a catheter showed significantly lower rectal doses and higher bladder doses than that with a catheter. To exclude artificial factors, such as rectal air, to account for this difference, we focused on the very short time interval from the first CT scanning to the second scanning. We also confirmed the almost total lack of a difference in the outline of the rectum using the image blending function in Variseed, with the exception of a slight difference in the rectal position due to rectal peristaltic motion.

During brachytherapy, a small change in the distance between the source and tissues can result in a meaningfully large difference in dose distribution. In this study, the mean source location difference in 1,200 sources among 18 patients who underwent the procedure with and without a catheter was $1.3 \mathrm{~mm}$. These displacements of the seeds with respect to spatial direction were systematic rather than random, as presented in Figure 2. The $R D_{2 c c}$ and $R V_{100}$ values were significantly different on $\mathrm{CT}$ with versus without the catheter $\left(\mathrm{RD}_{2 \mathrm{cc}}: 93.4 \mathrm{~Gy}\right.$ vs. $85.1 \mathrm{~Gy}, p<0.001 ; \mathrm{RV}_{100}$ : $0.42 \mathrm{cc}$ vs. $\left.0.21 \mathrm{cc}, p<0.001\right)$. $\mathrm{BD}_{1 \mathrm{cc}}$ values determined on $\mathrm{CT}$ were significantly lower with than without the catheter $\left(\mathrm{BD}_{1 \mathrm{cc}}: 75.3 \mathrm{~Gy}\right.$ vs. $81.2 \mathrm{~Gy}$, $p=0.027)$. A catheter placed in the prostatic urethra can produce a source shift and change the rectal and bladder doses. Even a smaller diameter urethral catheter $(8 \mathrm{Fr}$, 
Table 1. The estimated prostate volumes and results of the analysis of dose-volume histograms (DVHs) with and without the catheter

\begin{tabular}{|c|c|c|c|c|c|c|}
\hline & \multicolumn{2}{|c|}{ With catheter } & \multicolumn{2}{|c|}{ Without catheter } & \multirow[t]{2}{*}{ Mean difference ${ }^{1}$} & \multirow[t]{2}{*}{$p$ value } \\
\hline & Mean & Range & Mean & Range & & \\
\hline Prostate & - & - & - & - & - & - \\
\hline Volume (ml) & 23.8 & $12.6-38.4$ & 24 & $12.8-39.2$ & -0.2 & 0.002 \\
\hline $\mathrm{D}_{90}(\mathrm{~Gy})$ & 163.6 & $117.3-188.5$ & 161.6 & $114.9-184.3$ & 2 & 0.012 \\
\hline $\mathrm{V}_{100}(\%)$ & 94.6 & 79.6-99.6 & 93.9 & $78.9-99.5$ & 0.7 & 0.023 \\
\hline Urethra & - & - & - & - & - & - \\
\hline $\mathrm{UD}_{10}(\%)$ & 152 & $122.5-189.5$ & 156.5 & $131.9-191.2$ & -4.5 & $<0.001$ \\
\hline $\mathrm{UD}_{30}(\%)$ & 139.3 & $119.1-170.0$ & 142.7 & $125.4-170.9$ & -3.4 & 0.001 \\
\hline Rectum & - & - & - & - & - & - \\
\hline $\mathrm{RD}_{2 \mathrm{Cc}}$ (Gy) & 93.4 & $74.0-116.0$ & 85.1 & $66.0-114.1$ & 8.3 & $<0.001$ \\
\hline $\mathrm{RV}_{100}(\mathrm{cc})$ & 0.42 & $0.01-1.112$ & 0.21 & $0-1.06$ & 0.21 & $<0.001$ \\
\hline Bladder & - & - & - & - & - & - \\
\hline $\mathrm{BD}_{1 \mathrm{cc}}(\mathrm{Gy})$ & 75.3 & $43.5-102.6$ & 81.2 & $45.4-119.7$ & -5.9 & 0.027 \\
\hline
\end{tabular}

${ }^{1}$ Comparisons between with and without catheter, $D_{90}$ - the minimum dose received by $90 \%$ of the prostate volume, $V_{100}$ - the percent volume of the post-implant prostate receiving $100 \%$ of the prescribed dose, $U D_{10}$ and $U D_{30}$ - the minimum doses received by $10 \%$ and $30 \%$ of the urethral volume, $R D_{2 c c}-$ the minimum dose received by $2 \mathrm{cc}$ of the rectum, $R V_{100}$ - the rectal volume in cubic centimeters that received $>100 \%$ of the prescribed dose, $B D_{1 c c}-$ the minimum dose received by 1 cc of the bladder
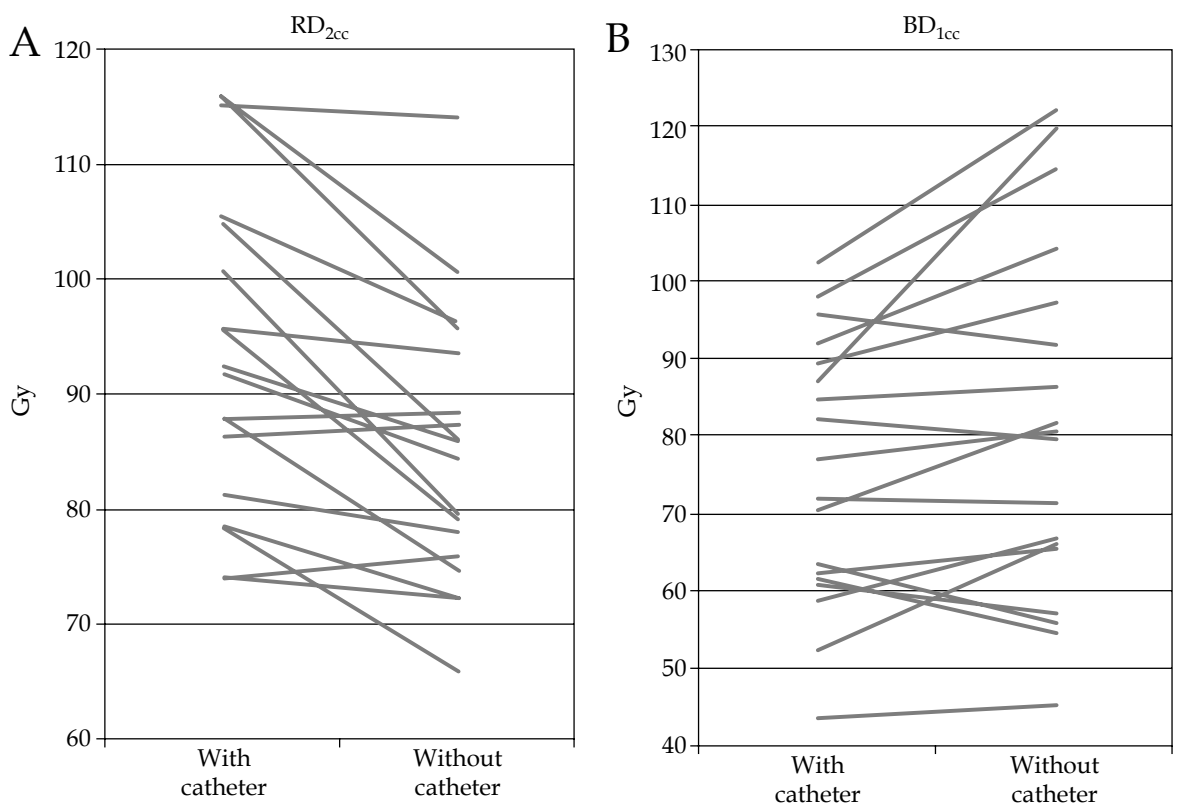

$B D_{1 c c}$ - minimum dose received by $1 c c$ of the bladder, $C T$ - computed tomography, $P I D$ - post-implant dosimetry, $R D_{2 c c}-$ minimum dose received by $2 c c$ of the rectum

Fig. 3. $R D_{2 c c}(A)$ and $B D_{1 c c}(B)$ values in each patient with or without a catheter. The $\mathrm{RD}_{2 c c}$ value $(93.4 \mathrm{~Gy})$ on $C T$ with a catheter was significantly higher than that $(85.1 \mathrm{~Gy})$ without a catheter $(p<0.001)$. The $\mathrm{BD}_{1 \mathrm{cc}}$ value $(75.3 \mathrm{~Gy})$ on $\mathrm{CT}$ with a catheter was significantly lower than that $(81.2 \mathrm{~Gy})$ without a catheter $(p=0.027)$

$2.7 \mathrm{~mm}$ ) provided a significant difference in the dose distribution in this study.

Previous reports have shown that PID performed with a urethral catheter results in differences in dosimetric parameters for the prostate and urethra. Brezovich et al. showed that post-implant images taken with a urethral catheter in place result in lower urethral doses than those actually delivered [22]. Shirvani et al. also reported that urinary catheterization artificially reduces target coverage in PID [23]. Brezovich et al. evaluated source positions and urethral doses on transrectal ultrasound images, and Shirvani et al. assessed prostate dose coverage on CT im- 
ages. In this study, we used CT images to more easily and accurately identify all seed positions compared with those on ultrasound images, and showed that a urinary catheter would change the dose volume parameter to the rectum and bladder. There are few reports evaluating the changes in seed distribution on CT and the dosimetric differences in the rectum and bladder doses, because of the use of a urinary catheter. Our recommended method using the two sets of CT images is a substantiated technique for demonstrating the actual post-implant irradiation dose. Because our recommended methodology can be performed easily and more truly demonstrates the real situation; i.e., that prostate cancer patients are irradiated without a urinary catheter after implantation, it will be preferred. We compared the prostate coverage $\left(D_{90}\right.$ and $V_{100}$ ) with and without the catheter. $D_{90}$ and $V_{100}$ values were significantly different on $\mathrm{CT}$ with versus without the catheter $\left(\mathrm{D}_{90}: 164 \mathrm{~Gy}(95 \%)\right.$ vs. $162 \mathrm{~Gy}(94 \%), p=0.012$; $\mathrm{V}_{100}: 113 \%$ vs. $\left.111 \%, p=0.023\right)$. Although the mean $\mathrm{D}_{90}$ and $\mathrm{V}_{100}$ values on PID without a catheter were significantly different from those on PID with a catheter, the differences were slight (2 Gy and $0.7 \%$, respectively) (Table 1 ). The influence of this very minute difference in a clinical setting may be ignored. These results suggest that the seeds move together with the target. The $\mathrm{UD}_{10}$ and $\mathrm{UD}_{30}$ values were higher than those on PID with a catheter. It was supposed that these differences were because seeds move toward the urethra upon catheter removal. The urethra on PID without a catheter was contoured as the same diameter structure as the urethra on CT images with a catheter in this study because the contouring of the prostatic urethra on PID without a catheter remains unknown. In the case of permanent prostate brachytherapy after transurethral resection [24], the results may be different because of urethral distension. As a next step, it would be interesting to quantitatively examine not only the urethral dose but also the rectum and bladder doses.

One limitation of the present study is the possible influence of inter- and intra-observer contouring, although the outlines of the rectum and bladder on CT are clear. Furthermore, there might be source reconstruction uncertainties using the automatic seed finding function with manual adjustments. Source reconstruction uncertainties may be indicated in the head-tail direction because they depend on the CT slice thickness. Therefore, the bladder dose can be affected, particularly by source reconstruction uncertainties.

Another fundamental limitation of the present study is that, although the catheters were removed with great care, the systematic displacement of the seeds may be due to the patients not being randomized. If the patients are scanned first without the catheter followed by scanning with catheter, the results might be different. It might be necessary for the patients to be randomized to the groups scanned first without the catheter and to the groups scanned first with the catheter. However, after the first CT scanning without the catheter, it will be difficult to insert the catheter into the urethra without moving the body and to perform the second CT scanning with the catheter in the same position.
Another limitation is that further investigations are necessary to determine whether combining post-implant assessments with and without a catheter influences the occurrence of late toxicity. If such an influence is demonstrated, more detailed information regarding the bladder and rectal dose constraints will be available to predict the occurrence of late bladder and rectal toxicity. Previous studies have attempted to suggest a relationship between dosimetric parameters and post-implant late toxicities [25-27]. Although comparisons between studies are difficult due to variations in the dosimetric parameters, previous studies have shown a greater association between higher normal tissue doses and late toxicities. Snyder et al. [25] demonstrated rectal complications to be directly related to the volume of the rectum receiving the prescribed dose after I-125 brachytherapy alone. The prescribed dose (160 Gy) delivered to $<1.3 \mathrm{cc}$ of rectal tissue resulted in a $5 \%$ rate of proctitis at 5 years vs. $18 \%$ for volumes $>1.3 \mathrm{cc}$. Kalakota et al. [26] reported that men with the volume of the rectum receiving the prescribed dose $\geq 0.05$ cc had a $26 \%$ risk of Grade $2+$ toxicity compared with $0 \%$ for the volume of the rectum receiving the prescribed dose $<0.05$ cc with I-125 brachytherapy after EBRT. An accurate dose assessment is important to demonstrate that complications are directly related to the volume of the organ at risk receiving the prescribed dose after I-125 implantation, and to provide more detailed information to allow the prediction of late bladder and rectal toxicity.

\section{Conclusions}

Post-implant dosimetry without a catheter showed significantly lower rectal doses than PID with a catheter. We recommend the PID procedure on CT images without a catheter, and the use of CT with a catheter to identify the urethral position.

\section{Disclosure}

Authors report no conflict of interest.

\section{References}

1. Ragde H, Blasko JC, Grimm PD et al. Interstitial iodine- 125 radiation without adjuvant therapy in the treatment of clinically localized prostate carcinoma. Cancer 1997; 80: 442-453.

2. Wallner K, Roy J, Harrison L. Tumor control and morbidity following transperineal iodine 125 implantation for stage T1/T2 prostatic carcinoma. J Clin Oncol 1996; 14: 449-453.

3. Grimm P, Billiet I, Bostwick D et al. Comparative analysis of prostate-specific antigen free survival outcomes for patients with low, intermediate and high risk prostate cancer treatment by radical therapy. Results from the Prostate Cancer Results Study Group. BJU Int 2012; 109: 22-29.

4. Aronowitz JN, Rivard MJ. The phylogeny of permanent prostate brachytherapy. J Contemp Brachytherapy 2013; 5: 89-92.

5. Kubicek GJ, Naguib M, Redfield S et al. Combined transperineal implant and external beam radiation for the treatment of prostate cancer: a large patient cohort in the community setting. Brachytherapy 2011; 10: 449-453.

6. Moerland MA, Wijrdeman HK, Beersma R et al. Evaluation of permanent I-125 prostate implants using radiography and magnetic resonance imaging. Int J Radiat Oncol Biol Phys 1997; 37: 927-933. 
7. Tanaka O, Hayashi S, Matsuo M et al. Comparison of MRI based and CT MRI fusion based postimplant dosimetric analysis of prostate brachytherapy. Int J Radiat Oncol Biol Phys 2006; 66: 597-602.

8. Polo A, Cattani F, Vavassori A et al. MR and CT image fusion for postimplant analysis in permanent prostate seed implants. Int J Radiat Oncol Biol Phys 2004; 60: 1572-1579.

9. Smith WL, Lewis C, Bauman G et al. Prostate volume contouring: A 3D analysis of segmentation using 3DTRUS, CT, and MR. Int J Radiat Oncol Biol Phys 2007; 67: 1238-1247.

10. Willins J, Wallner K. CT-based dosimetry for transperineal I-125 prostate brachytherapy. Int J Radiat Oncol Biol Phys 1997; 39: 347-353.

11. Gay HA, Barthold HJ, O'Meara E et al. Pelvic normal tissue contouring guidelines for radiation therapy: a Radiation Therapy Oncology Group consensus panel atlas. Int J Radiat Oncol Biol Phys 2012; 83: 353-362.

12. Stock RG, Stone NN, Tabert A et al. A dose-response study for I-125 prostate implants. Int J Radiat Oncol Biol Phys 1998; 41: 101-108.

13. Nath R, Bice WS, Butler WM et al. AAPM recommendations on dose prescription and reporting methods for permanent interstitial brachytherapy for prostate cancer: report of Task Group 137. Med Phys 2009; 36: 5310-5322.

14. Crook JM, Potters L, Stock RG et al. Critical organ dosimetry in permanent seed prostate brachytherapy: defining the organs at risk. Brachytherapy 2005; 4: 186-194.

15. Ohashi T, Momma T, Yamashita $S$ et al. Dosimetric effects of prone and supine positions on post-implant assessments for prostate brachytherapy. J Contemp Brachytherapy 2013; 5: 122-126.

16. Merrick GS, Butler WM, Grimm P et al. Permanent prostate brachytherapy extracapsular radiation dose distributions: analysis of a multi-institutional database. I Contemp Brachytherapy 2013; 5: 117-121.

17. Maletz KL, Ennis RD, Ostenson J et al. Comparison of CT and MR-CT Fusion for Prostate Post-Implant Dosimetry. Int J Radiat Oncol Biol Phys 2012; 82: 1912-1917.

18. Vidakovic S, Jans HS, Alexander A et al. Post-implant computed tomography-magnetic resonance prostate image registration using feature line parallelization and normalized mutual information. J Appl Clin Med Phys 2006; 8: 21-32.

19. Tanaka O, Hayashi S, Sakurai K et al. Importance of the CTMRI fusion method as a learning tool for CT-based postimplant dosimetry in prostate brachytherapy. Radiother Oncol 2006; 81: 303-308.

20. Amdur RJ, Gladstone D, Leopold KA et al. Prostate seed implant quality assessment using MR and CT image fusion. Int J Radiat Oncol Biol Phys 1999; 43: 67-72.

21. Prestidge BR, Bice WS, Kiefer EJ et al. Timing of computed tomography-based postimplant assessment following permanent transperineal prostate brachytherapy. Int J Radiat Oncol Biol Phys 1998; 40: 1111-1115.

22. Brezovich IA, Pareek PN, Duan J et al. Effect of Foley catheters on seed positions and urethral dose in (125)I and (103) Pd prostate implants. Int J Radiat Oncol Biol Phys 2001; 49: 1461-1468.

23. Shirvani SM, Kudchadker RJ, Bruno TL et al. Impact of urinary catheterization on dosimetry after prostate implant brachytherapy with palladium-103 or iodine-125. Brachytherapy 2011; 10: 269-274.

24. Salembier C, Rijnders A, Henry A et al. Prospective multicenter dosimetry study of low-dose Iodine- 125 prostate brachytherapy performed after transurethral resection. J Contemp Brachytherapy 2013; 5: 63-69.

25. Snyder KM, Stock RG, Hong SM et al. Defining the risk of developing grade 2 proctitis following 125I prostate brachytherapy using a rectal dose-volume histogram analysis. Int J Radiat Oncol Biol Phys 2001; 50: 335-341.

26. Kalakota K, Rakhno E, Pelizzari CA et al. Late rectal toxicity after prostate brachytherapy: influence of supplemental external beam radiation on dose-volume histogram analysis. Brachytherapy 2010; 9: 131-136.

27. Nakamura R, Kikuchi K, Tanji S et al. Narrow safety range of intraoperative rectal irradiation exposure volume for avoiding bleeding after seed implant brachytherapy. Radiat Oncol 2012; 7: 15 